\title{
Entropic Approach and Evolution Strategies for Optimizing the Image Segmentation by Pixel Classification: Application to Quality Control
}

\author{
M. Merzougui, \\ LABO MATSI, ESTO, B.P 473, \\ University of Mohammed I, \\ OUJDA, MOROCCO.
}

\author{
M. Nasri \\ LABO MATSI, ESTO, B.P 473, \\ University of Mohammed I, \\ OUJDA, MOROCCO
}

\author{
B. Bouali \\ LABO AGA, FSO, \\ University of Mohammed I, \\ OUJDA, MOROCCO
}

\begin{abstract}
In this paper, a segmentation method based on pixel classification and evolution strategies is proposed. Before segmentation, the number of classes is determined by the principle of maximum entropy. The proposed approach is validated on some synthetic and real images and, it shows to be very interesting as decision support in quality control
\end{abstract}

\section{Keywords}

Segmentation, segmentation by pixel classification, evolutionary strategies, evolutionary segmentation, principle of maximum entropy.

\section{INTRODUCTION}

The segmentation is an essential stage in image processing. There are many consistent methods available today for image segmentation, among these, there is the segmentation based on pixels classification as a function of their grey level values [1][2][3][4]. Every pixel in the image holds an inherent relationship with the pixels in its surrounding. The information at a particular pixel may be in relation with the information over the whole or part of the image. The mean or median value of the gray level of the pixel is selected.

The process of segmentation by pixel classification consists of three stages: [1] [2] [3] [4].

- Acquisition of data for each pixel in order to form the attribute vector

- Estimation of the number of classes with the principle of maximum entropy.

- Pixel classification based on the acquired information

\section{$\checkmark \quad$ Acquisition stage}

For each pixel, two values are calculated, the mean value of the grey levels $(M G L)$, and the difference between the $M G L$ and the maximum value of the grey levels surrounding the particular pixel $(D M G L)$. For this purpose, a square window centred at the particular pixel is used.

\section{$\checkmark \quad$ Estimate the number of classes}

The principle of maximum entropy is used.

\section{$\checkmark \quad$ Classification stage}

Kmeans and evolutionary Kmeans are used for this classification purpose. The two algorithms use the information provided by the parameters $M G L$ and $D M G L$, associated with each individual pixel in order to classify the pixel with respect to centers that evolve at each iteration

In Section 2 image segmentation with Kmeans algorithm is presented. Section 3 gives an introduction to evolution strategies approach and the proposed evolutionary Kmeans algorithm along with the image evolutionary segmentation. The estimation of the number of classes with the principle of maximum entropy is presented in section 4 . In section 5 , a validation of our approach is given; experimental results are obtained over some synthetic and real images. Finally, a conclusion is given.

\section{KMEANS CLASSIFICATION}

\subsection{Descriptive elements}

Consider a set of $M$ objects $\left\{O_{1}, O_{2}, \ldots, O_{M}\right\}$ characterized by $N$ attributes, grouped in a line vector form $V=\left(\begin{array}{llll}a_{1} & a_{2} & \ldots & a_{N}\end{array}\right)$. Let $R_{i}=\left(a_{i j}\right)_{1 \leq j \leq N}$ be a line vector of $\boldsymbol{R}^{N}$ where $a_{i j}$ is the value of the attribute $a_{j}$ for the object $O_{i}$. Let mat_obs be a matrix of $M$ lines (representing the objects $O_{i}$ ) and $N$ columns (representing the attributes $a_{j}$ ):

$$
\text { mat_obs }=\left(a_{i j}\right)_{\substack{1 \leq i \leq M \\ 1 \leq j \leq N}}
$$

$V$ is the attribute vector, $R_{i}$ is the observation associated with $O_{i}$ or the realization of the attribute vector $V$ for this object, $\boldsymbol{R}^{N}$ is the observations space [1] and mat_obs is the observation matrix associated with $V$. The $\mathrm{i}^{\text {th }}$ line of mat_obs is the observation $R_{i}$. $R_{i}$ belongs to a class $C L_{s}, s=1, \ldots, \bar{C}$.

\subsection{Kmeans algorithm}

The Kmeans algorithm is one of the most common algorithms used for the classification, maxobs observations $\left(R_{i}\right)_{1 \leq i \leq \mathrm{M}}$ which must be associated with $C$ classes $\left(C L_{s}\right)_{1 \leq s \leq C}$ of centers $\left(\mathrm{g}_{s}\right)_{1 \leq s \leq C}$ are given. The centers $\left(\mathrm{g}_{s}\right)_{1 \leq s \leq C}$ are line vectors of $\mathrm{N}$ dimension.

The Kmeans is based on the minimization of the optimization criterion given by: [5] [6] [4]

$$
J=\frac{1}{2} \sum_{i=1}^{M} \sum_{s=1}^{C}\left\|R_{i}-g_{s}\right\|^{2}
$$

where $\|$.$\| is a distance which is generally supposed to be$ Euclidean.

The $K M$ algorithm supposes that the number of classes $C$ is known a priori. 


\subsection{Kmeans segmentation}

The objects that are processed by the $K M$ algorithm are the pixels of the input image. The observation matrix in this case is formed by two columns which represent the attributes associated with each pixel of the image: the columns are associated with the $M G L$ and the DMGL. The size of the square window used must have an odd length $(3 * 3,5 * 5 \ldots)$ [1][2][4].

In this process each pixel is attributed to a specific class. The resulting image is segmented into $\mathrm{C}$ different regions where each region corresponds to a class.

\section{EVOLUTION STRATEGIES}

Evolutionary strategies $(E S)$ are particular methods for optimizing functions. These techniques are based on the evolution of a population of solutions which under the action of some precise rules optimize a given behaviour, which initially has been formulated by a given specified function called fitness function [7][8].

An ES algorithm manipulates a population of constant size. This population is formed by candidate points called chromosomes. Each of the chromosomes represents the coding of a potential solution to the problem to be solved, it is formed by a set of elements called genes, and these are real.

At each iteration, called generation, is created a new population from its predecessor by applying the genetic operators: selection and mutation. The mutation operator perturbs with a Gaussian disturbance the chromosomes of the population in order to generate a new population permitting to further optimize the fitness function.

This procedure allows the algorithm to avoid the local optimums. The selection operator consists of constructing the population of the next generation. This generation is constituted by the pertinent individuals [6] [7][9].

Figure 1 illustrates the different operations to be performed in a standard ES algorithm [7][9] :

\section{Random generation of the initial population \\ Fitness evaluation of each chromosome \\ Repeat \\ Select the parents \\ Update the genes by mutation \\ Select the next generation \\ Fitness evaluation of each chromosome \\ Until Satisfying the stop criterion}

\section{Figure 1: Standard SE algorithm.}

\section{EVOLUTIONARY KMEANS}

\subsection{Proposed coding}

The $K M$ algorithm consists of selecting among all of the possible partitions the optimal partition by minimizing a criterion. This yields the optimal centers $\left(g_{s}\right)_{1 \leq s \leq C}$. Thus, the real coding following is suggested:

$$
\begin{aligned}
& c h r=\left(g_{s j}\right)_{1 \leq s \leq C, 1 \leq j \leq N} \\
& =\left(g_{11} . g_{1 N} g_{21} . g_{2 N} \cdot . g_{S 1} . g_{S N} \cdot g_{C 1} \cdot . g_{C N}\right)
\end{aligned}
$$

The chr chromosome is a real line vector of dimension $C \times N$. The genes $\left(g_{s j}\right)_{1 \leq j \leq N}$ are the components of the $g_{s}$ center:

$$
g_{s}=\left(g_{s j}\right)_{1 \leq j \leq N}=\left(g_{s 1} g_{s 2} \cdot g_{s j} \cdot g_{s N}\right)
$$

To avoid that the initial solutions be far away from the optimal solution, each chromosome $c h r$ of the initial population should satisfy the condition:

$$
g_{s j} \in\left[\min a_{i j 1 \leq i \leq M}, \max a_{i j 1 \leq i \leq M}\right]
$$

In the $E K M$ algorithm, any chromosome with a gene that does not satisfy this constraint is eliminated.. This gene, if any, is replaced by another one which complies with the constraint [8].

\subsection{The proposed fitness function}

Let $c h r$ be a chromosome of the population formed by the centers $\left(\mathrm{g}_{s}\right)_{1 \leq s \leq C}$, for computing the fitness function value associated with chr, fitness function $\mathrm{F}$ which expresses the behavior to optimize (criterion $\mathrm{J}$ ) is defined.:

$$
F(\text { chr })=\frac{1}{M} \sum_{i=1}^{M} \sum_{s=1}^{C}\left\|R_{i}-g_{s}\right\|^{2}
$$

The chromosome chr is optimal if $\mathrm{F}$ is minimal.

\subsection{The proposed mutation operator}

The performances of an algorithm based on evolutionary strategies are evaluated according to the mutation operator used [6]. One of the mutation operator form proposed in the literature [10] [11] is given by:

$$
c h r^{*}=c h r+\sigma \times N(0,1)
$$

where $c h r^{*}$ is the new chromosome obtained by a Gaussian perturbation of the old chromosome chr. $N(0,1)$ is a Gaussian disturbance of mean value 0 and standard deviation value $1, \sigma$ is the strategic parameter. $\sigma$ is high when the fitness value of $c h r$ is high. When the fitness value of $c h r$ is low, $\sigma$ must take very low values in order to be not far away from the global optimum.

Of this approach, a new shape of the operator of the mutation is proposed. The fact to propose a new operator of the mutation is motivated by the interest to reach the global solution in a small computational time.

Let $c h r$ be a chromosome of the population formed by the centers $\left(g_{s}\right)_{1 \leq s \leq C}$.

Let $R_{i} \in C L_{s}$ if $\left\|R_{i}-g_{s}\right\|=\min _{s^{\prime}=1, C}\left\|R_{i}-g_{s^{\prime}}\right\|$, i.e. the

class consisting of the $\mathrm{R}_{\mathrm{i}}$ observations that are closest to the center $\mathrm{g}_{\mathrm{s}}$. Let $\mathrm{g}_{\mathrm{s}}^{\circ}$ be the center of gravity of the $\mathrm{CL}_{\mathrm{s}}$ class

$$
g_{S}^{\circ}=\frac{\sum_{i} \in C L_{s}}{l_{S}} \text { where } l_{s}=\operatorname{card}\left(C L_{S}\right)
$$

The mutation operator proposed in this work consists in generating, from the chr, the new chromosome $c h r^{*}$ formed by the centers $\left(g *_{s}\right)_{1 \leq s \leq \mathrm{C}}$, as:

$$
g{ }_{s}=g_{s}+f_{m} \times\left(g^{\circ}{ }_{s}-g_{s}\right) \times N(0,1)
$$

where $f_{m}$ is a multiplicative constant factor taken to be randomly chosen between 0.5 and 1 . The new strategic parameter proposed $\sigma^{\prime}=f_{m} \times\left(g^{\circ}{ }_{s}-g_{s}\right)$ is low when $g_{s}$ gets closer to $\mathrm{g}_{\mathrm{s}}^{\circ}$ and is high when $\mathrm{g}_{\mathrm{s}}$ is far from $\mathrm{g}_{\mathrm{s}}{ }_{\mathrm{s}}$. The proposed $\sigma^{\prime}$ has two advantages:

- When chr is far from the global solution, chr is subjected to a strong Gaussian perturbation allowing 
chr to move more quickly in the research space and in the same time to avoid local solutions.

- $\sigma^{\prime}$ controls the Gaussian perturbation level. Indeed, as the chromosome $\mathrm{chr}$ gets closer to the global solution, the Gaussian perturbation level is reduced until becoming null at convergence.

Generating children from parent chromosomes the technique of selection by ordering is adopted. The elitist technique is also used [11].

\subsection{The proposed EKM algorithm}

Figure 2 shows the different steps of the proposed $E K M$ algorithm. [5][6] [12] [13].

Stage 1:
1.1. Fix:
- The size of the population maxpop.
- The maximum number of generations maxgen.
- The number of classes $C$.
1.2. Generate randomly the population $P$ :
$\quad P=\{$ chrl, .., chrk, .., chrmaxpop $\}$
1.3. Verify for each chr of $P$ the constraint:

1.3. Verify for each $c h r$ of $P$ the constraint: $g_{s j} \in\left[\min a_{i j}, \max a_{i j}\right], 1 \leq i \leq M$

1.4. For each $c h r$ of $P$ attribute, the observations $R i$ to the corresponding classes:

$$
R_{i} \in C L_{s} \text { if }\left\|R_{i}-g_{s}\right\|=\min _{s^{\prime}=1, C}\left\|R_{i}-g_{s^{\prime}}\right\|
$$

1.5. Update the population $P$, for each $c h r$ of $P$ do:

$$
g_{S}^{\prime}=\frac{g_{s}+\sum_{R_{i} \in C L_{s}} R_{i}}{1+l_{S}} \text { where } l_{S}=\operatorname{card}\left(C L_{S}\right)
$$

1.6. Compute for each $c h r$ of $P$ its fitness value $F(c h r)$.

Stage 2:

\section{Repeat}

2.1. Order the chromosomes $c h r$ in $P$ from the best to the poor ( in an increasing order of $F$ ).

2.2. Choose the best chromosomes chr.

2.3. For each $c h r$ of $P$ attribute, the observations $R i$ to the corresponding classes:

$$
R_{i} \in C L_{s} \text { if }\left\|R_{i}-g_{s}\right\|=\min _{s^{\prime}=1, C}\left\|R_{i}-g_{s^{\prime}}\right\|
$$

2.4. Generate randomly the constant $f_{m}\left(f_{m} \in[0.5,1]\right)$.

2.5. Mutation of all the chromosomes $c h r$ of $P$ except the first one (elitist technique):

$$
g *_{s}=g_{s}+f_{m} \times\left(g_{s}^{\circ}-\mathrm{g}_{\mathrm{s}}\right) \times N(0,1)
$$

2.6. For each $c h r$ of $P$ attribute except the first one, the observations $R i$ to the corresponding classes:

$$
R_{i} \in C L_{s} \text { if }\left\|R_{i}-g_{s}\right\|=\min _{s^{\prime}=1, C}\left\|R_{i}-g_{s^{\prime}}\right\|
$$

2.7. Update the population $P$, for each $c h r$ of $P$ except for the first one, do:

$$
g_{S}^{\prime}=\frac{g_{s}+\sum_{R_{i} \in C L_{s}} R_{i}}{1+l_{s}} \text { where } l_{S}=\operatorname{card}\left(C L_{S}\right)
$$

(The population $P$ obtained after the updating is the population of the next generation )

2.8. Compute for each $c h r$ of $P$ its fitness value $F(c h r)$.

Until $N b \_g e n$ (generation number) ! maxgen

\subsection{EKmeans Segmentation}

The procedure to be carried out here is exactly the same at that taken in (2.3) for the Kmeans segmentation. The difference is in the classification algorithm which now is evolutionary.

\section{DETERMINATION OF THE OPTIMAL NUMBER OFCLASSES}

\subsection{Principle of maximum entropy}

Choosing the right number of classes $C$, in many partition problems, is a difficult task. Several criteria for choosing the optimal number of classes, based on different approaches, have been proposed in the literature [14]. In this paper the principle of maximum entropy is retained such as:

An entropy measure of information provided by all classes is defined by: [14]

where :

$$
S=-\sum_{j=1}^{C} \sum_{i \in C L j}\left(\frac{P_{i j}}{C}\right) \ln \left(\frac{P_{i j}}{C}\right)
$$

$$
S=-\frac{1}{C} \sum_{j=1}^{C} \sum_{i \in C L j} P_{i j} \ln \left(P_{i j}\right)+\ln (C)
$$

i.e:

with :

$$
S=\frac{1}{C} \sum_{j=1}^{C} S_{j}+\ln (C)
$$

$$
S_{j}=-\sum_{i \in C L j} P_{i j} \ln \left(P_{i j}\right)
$$

$S_{j}$ is the entropy corresponding to class $j$. The optimal number of classes for which the $\mathrm{C}_{\mathrm{opt}}$ is the entropy $\mathrm{S}$ is maximum [14].

Coefficients $P_{i j}$ (probabilities are the links between points $i$ and their class $C_{j}$ center $g_{j}$ ) are given by:

$$
P_{i j}=\frac{\exp \left[-C\left\|x_{i}-g_{j}\right\|^{2}\right\rfloor}{\sum_{i \in C L j} \exp \left[-C\left\|x_{i}-g_{j}\right\|^{2}\right]}
$$

Finally, our criterion is defined as entropy:

$$
\mathrm{M}_{\mathrm{ENP}}=\frac{1}{C} \sum_{j=1}^{C} S j+\ln (C)
$$

Where $S_{j}$ is defined by equation (13) that uses $P_{i j}$ defined in equation (14). The optimal number of classes will $\mathrm{C}_{\text {opt }}$ one for which the maximum value is $M_{E N P}$ [14] [15] [16].

$M_{E N P}$ algorithm is executed for several values of $\mathrm{C}, \mathrm{C} \in\left[C_{m i n}\right.$, $\left.C_{\max }\right] \quad\left(2 \leq C_{\min }\right.$ et $\left.C_{\max }<<M\right)$. For each value of $\mathrm{C}$, this algorithm gives the convergence $f_{M E N P}(\mathrm{C})$. The optimal number of classes is $\mathrm{C}_{\mathrm{opt}}$ whose value $f_{M E N P}(\mathrm{C})$ is maximum.

\section{EXPERIMENTAL RESULTS AND EVALUATIONS}

\subsection{Introduction}

In order to evaluate the performances of the proposed method, three grey level images are considered, a synthesised image, and two real images [1]. The segmentation is carried out by $K M$ and $E K M$ algorithms after that $\mathrm{C}_{\text {opt }}$ has been obtained by maximum entropy principle.

Figure 2: The proposed EKM algorithm. 


\subsection{Synthetic image}

A synthetic image is constructed that is named SYNTH1 of size $143 * 122$ (figure 3 ).

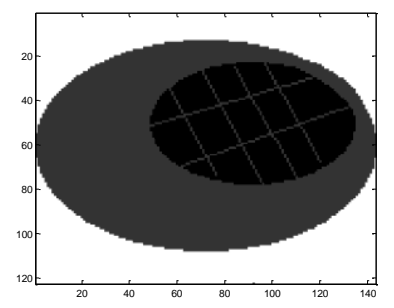

Figure 3: Synthesised image SYNTH1

Table 1 shows the classes of SYNTH1 along with the grey level values and the number of pixels in each class.

Table 1: Information on SYNTH1

\begin{tabular}{|c|c|c|c|}
\hline $\begin{array}{c}\text { Class } \\
\text { Region }\end{array}$ & description & $\begin{array}{c}\text { Level of } \\
\text { gray }\end{array}$ & $\begin{array}{c}\text { Number } \\
\text { of pixels }\end{array}$ \\
\hline 1 & background & 255 & 6755 \\
\hline 2 & $\begin{array}{c}\text { Big oval part } \\
\text { included lines) }\end{array}$ & 60 & 7339 \\
\hline 3 & Small oval part & 0 & 3352 \\
\hline
\end{tabular}

For this test the attributes $(M G L, D M G L)$ are considered in a 3 * 3 window. With the maximum entropy function, the values shown in table 2 and figure 4 are gotten:

Table 2: $f_{M E N P}$ for different values of $\mathrm{C}$

\begin{tabular}{|c|c|c|c|c|}
\hline $\mathbf{C}$ & $\mathbf{3}$ & $\mathbf{4}$ & $\mathbf{5}$ & $\mathbf{6}$ \\
\hline $\boldsymbol{f}_{\text {MENP }}$ & 0.4196 & 0.4129 & 0.3559 & 0.4008 \\
\hline
\end{tabular}

The maximum is achieved for $\mathrm{C}=3$, so $\mathrm{C}_{\mathrm{op}}=3$.

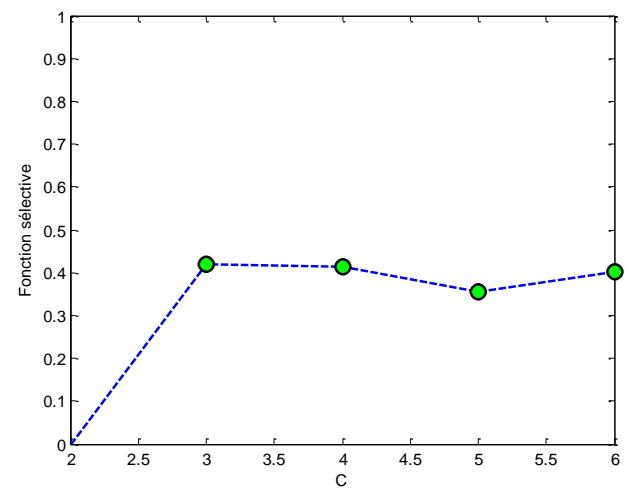

Figure 4 : Evolution of $f_{M E N P}$ function of $\mathrm{C}$,

$$
\mathrm{C}_{\text {opt }}=3 \text {. }
$$

One notices that the number of classes gotten by the algorithm of the entropy maximum coincides precisely with the real number of the classes.

\section{$\underline{\text { SYNTH1 image segmentation with } \mathrm{C}=3}$}

Figure 5 shows the results of segmentation by $K M$ and $E K M$ algorithms (three successive trials).
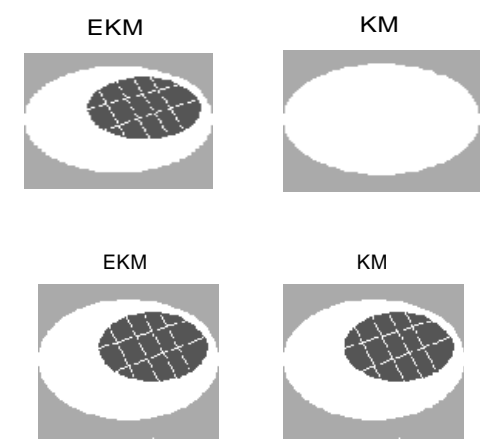

EKM

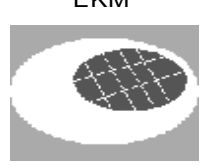

$\mathrm{KM}$

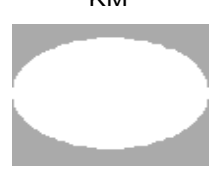

Figure 5 : Result of image segmentation

The EKM algorithm converges quickly in 2 generations as shown in figure 6 .

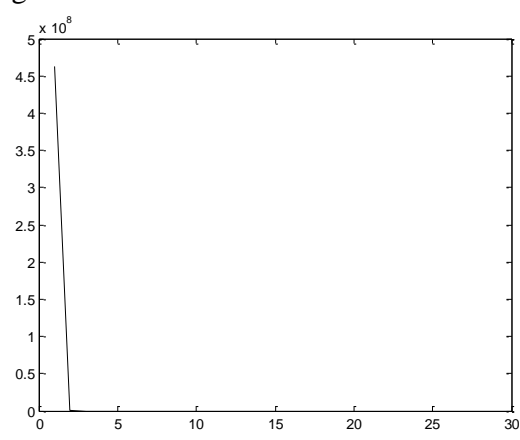

Figure 6: Fitness value with respect to the generation. Synth 1 image segmentation results are summarised in table 3.

Table 3: results of segmentation for Synth1 image

\begin{tabular}{|c|c|c|c|c|c|c|}
\hline \multirow{2}{*}{ description } & \multicolumn{3}{|c|}{ EKM } & \multicolumn{3}{c|}{ KM } \\
\cline { 2 - 7 } & Trial1 & Trial2 & Trial3 & Trial1 & Trial2 & Trial3 \\
\hline background & 6755 & 6755 & 6755 & 12410 & 6755 & 12410 \\
\hline $\begin{array}{c}\text { Big oval part } \\
\text { (included lines) }\end{array}$ & 7339 & 7339 & 7339 & 0 & 7339 & 0 \\
\hline $\begin{array}{c}\text { Small oval } \\
\text { part }\end{array}$ & 3352 & 3352 & 3352 & 5855 & 3352 & 5855 \\
\hline
\end{tabular}

The results show that the EKM clearly detects the all objects of the image, background, big oval part, small oval part and lines. The $K M$ was not able to detect the small oval part and lines.

The two algorithms were run several times and the EKM obtains each time the same result while the $K M$ obtains different results. We can conclude that the $E K M$ is the most stable; it outperforms the $K M$ and obtains good results.

\subsection{Blister pads image}

In this phase of testing, an image of blister pads of size $79 * 81$ which contains 10 stamps of longitudinal shape is taken, figure 7 . The objective is to check whether there is any tablet in the pad lacking or damaged. The window that is considered is of size $3 * 3$. 


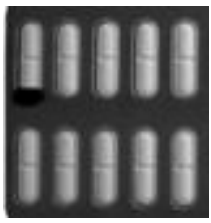

Figure 7: The original image

With the maximum entropy function the values shown in table 4 and figure 8 are gotten:

Table 4: $f_{M E N P}$ for different values of $\mathrm{C}$

\begin{tabular}{|c|c|c|c|c|}
\hline $\mathrm{C}$ & 3 & 4 & 5 & 6 \\
\hline$f_{\text {MENP }}$ & 0.2220 & 0.4129 & 0.3559 & 0.4008 \\
\hline
\end{tabular}

The maximum is achieved for $\mathrm{C}=4$, so $\mathrm{C}_{\mathrm{op}}=4$.

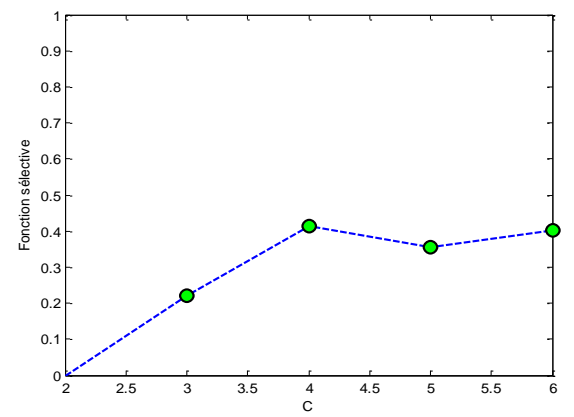

Figure 8 : Evolution of $f_{M E N P}$ function of $C, C_{\text {opt }}=4$.

\section{Pad image segmentation with $\mathrm{C}=4$}

The segmentation results in this case are shown in figure 9 for three different running of the $K M$ and $E K M$ algorithms. The result show that $E K M$ clearly detects the damage accruing on one of the tablets for the three running while the $K M$ fails to detect the defect for each.
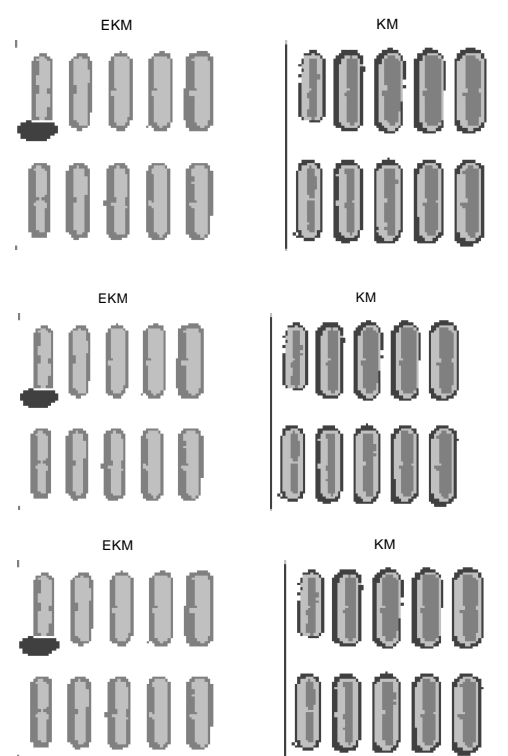

Figure 9: Results of image segmentation
Table 5: number of pixels for any class

\begin{tabular}{|c|c|c|c|c|}
\hline & $\begin{array}{c}\text { Class } \\
\text { number }\end{array}$ & Trial 1 & Trial 2 & Trial 3 \\
\hline \multirow{4}{*}{ EKM } & CL1 & 92 & 92 & 92 \\
& CL2 & 1216 & 1216 & 1216 \\
& CL3 & 1317 & 1317 & 1317 \\
& CL4 & 3774 & 3774 & 3774 \\
\hline \multirow{4}{*}{$K M$} & CL1 & 984 & 988 & 984 \\
& CL2 & 1161 & 1161 & 1161 \\
& CL3 & 1184 & 1184 & 1184 \\
& CL4 & 3070 & 3066 & 3070 \\
\hline
\end{tabular}

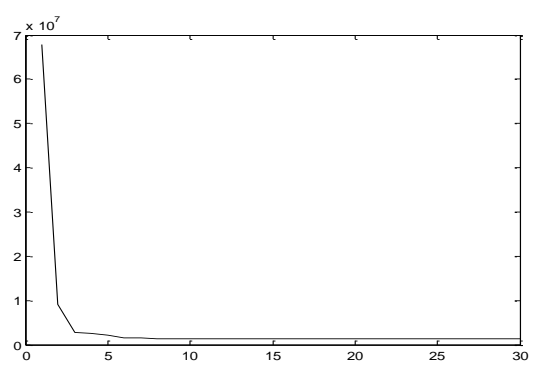

Figure 10 : Fitness value with respect to the generation.

Figure 10 shows the convergence of EKM algorithm, convergence is achieved very quickly, in three iterations, table 5 shows the results obtained by $K M$ and $E K M$ algorithms with details obtained on the classes for each running test. The $E K M$ is more stable and has outperformed the $K M$ algorithm.

\subsection{Small disc image}

In this phase of testing, an image of small disk of size 49 * 270 (figure 11) is taken. The objective is to detect the crack on the small disk. The window that is considered is of size $3 *$ 3.

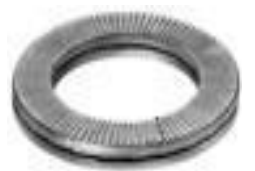

Figure11: The original image

With the maximum entropy function the values shown in table 6 and figure 12 are gotten:

Table 6: $f_{M E N P}$ for different values of $\mathrm{C}$

\begin{tabular}{|c|c|c|c|c|}
\hline $\mathrm{C}$ & 3 & 4 & 5 & 6 \\
\hline$f_{\text {MENP }}$ & 0.6718 & 0.4652 & 1.1619 & 1.0328 \\
\hline
\end{tabular}

The maximum is achieved for $\mathrm{C}=5$, so $\mathrm{C}_{\mathrm{op}}=5$.

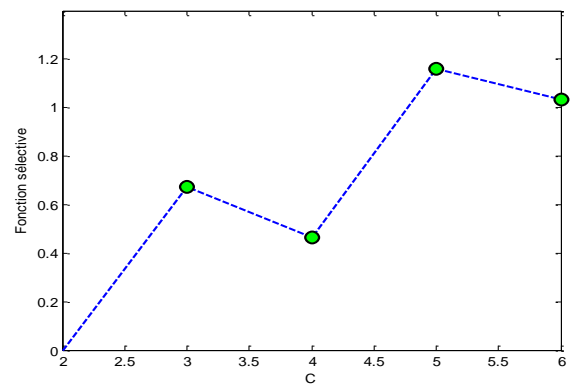

Figure 12: Evolution of $f_{M E N P}$ function of $C, C_{\text {opt }}=5$. 
Small disk image segmentation with $\mathrm{C}=5$

The segmentation results in this case are shown in figure 13 for three different running of the $K M$ and $E K M$ algorithms. The result show that $E K M$ clearly detects the crack on the disk for the three running while the $K M$ fails to detect the defect.
EKM

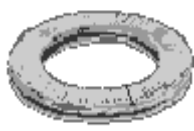

EKM

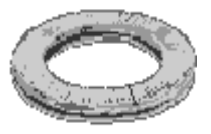

EKM

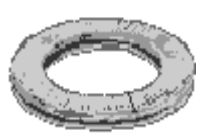

KM

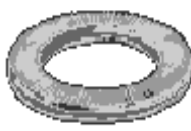

KM

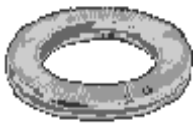

KM

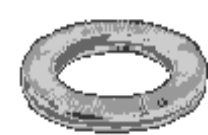

Figure 13 : Results of image segmentation

Table 7 : number of pixels for any class

\begin{tabular}{|c|c|c|c|c|}
\hline & $\begin{array}{c}\text { Class } \\
\text { number }\end{array}$ & Trial 1 & Trial 2 & Trial 3 \\
\hline \multirow{5}{*}{ EKM } & CL1 & 240 & 240 & 240 \\
& CL2 & 418 & 418 & 418 \\
& CL3 & 578 & 578 & 578 \\
& CL4 & 1320 & 1320 & 1320 \\
& CL5 & 1854 & 1854 & 1854 \\
\hline \multirow{5}{*}{ KM } & CL1 & 327 & 327 & 327 \\
& CL2 & 400 & 400 & 400 \\
& CL3 & 880 & 880 & 880 \\
& CL4 & 998 & 998 & 998 \\
& CL5 & 1805 & 1805 & 1805 \\
\hline
\end{tabular}

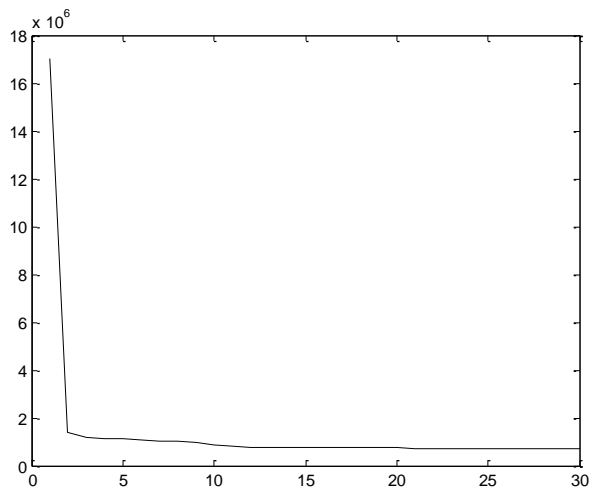

Figure 14 : Fitness value with respect to the generation.

Figure 14 shows the convergence of EKM algorithm, convergence is achieved very quickly, table 7 shows the results obtained by $K M$ and $E K M$ algorithms with details obtained on the classes for each running test. The EKM is more stable and has outperformed the $K M$ algorithm.

\section{CONCLUSION}

Kmeans image segmentation shows some stability difficulties due to the initialisation problem. The evolutionary Kmeans image segmentation is proposed in order to get around this difficulty. The proposed approach has been validated on synthetic and real images.

The experimental results obtained show the rapid convergence and the good performance of this approach. The instability problem has been eliminated.

The principle of maximum entropy is used to correctly estimate the optimal number of classes.

This approach may be used for problems of decision support in quality control.

\section{REFERENCES}

[1] Cocquerez T.P. et Phillip S. "Analyse d'images : Filtrage et segmentation". Editions MASSON, Paris, 1995.

[2] N.Vandenbroucke, L.Macaire, J.G. Postaire, Color image segmentation by pixel classification in an adapted hybrid color space. Application to soccer image analysis., Computer Vision and Image Understanding 90 (2), 190-216, 2003

[3] Christophe Saint-Jean, Classification paramétrique robuste partiellement supervisée en reconnaissance des formes, Thèse de Doctorat, université de La Rochelle UFR Sciences Laboratoire d'Informatique et d'Imagerie Industrielle 2001.

[4] L. Macaire, N. Vandenbroucke, J.G Postaire Segmentation d'images par classification spatiocolorimétrique des pixels. Traitement du signal vol 21 N spécial L'image numérique couleur 423-437, 2004.

[5] Nasri M. Contribution à la classification de données par Approches Evolutionnistes : Simulation et Application aux images de textures". Thèse de doctorat. Université Mohammed premier Oujda 2004

[6] Nasri M, M. EL Hitmy, H. Ouariachi and M.Barboucha. Optimization of a fuzzy classification by evolutionary strategies. In proceedings of SPIE Conf., 6 th international conference on quality control by artificial vision, vol. 5132, pp. 220230, USA,. Repulished as an SME Technical Paper by The society of manufacturing, 2003.

[7] Hall, L. Q., Özyurt, I. B. et Bezdek, J. C. Clustering with a genetically optimized approach ". IEEE Trans. on Evolutionary Computation, Vol.3, №2, pp. 103-110, july 1999.

[8] H.Ouariachi, Classification non -Supervisée de données par les réseaux de neurones et par une approche évolutionniste : application à la segmentation d'images. Thèse de doctorat. Université Mohammed premier Oujda 2001.

[9] Presberger, T., Koch, M. "Comparison of evolutionary strategies and genetic algorithms for optimization of a fuzzy controller". Proc. of EUFIT'95, Aachen, Germany, august 1995.

[10] Bezdek, J. C. "Cluster validity with fuzzy sets". J. Cybernetics, Vol.3, N³, pp. 58-73, 1974.

[11] Karayiannis, N. B., Bezdek, J. C. et Hathaway, R. J. "Repairs to GLVQ: a new family of competitive learning schemes". IEEE Trans. on Neural Networks, Vol.7, N5, pp. 1062-1071, 1996. 
[12] A. EL Allaoui, M. Merzougui, M. Nasri, M. EL Hitmy and H. Ouariachi. Optimization of Unsupervised Classification by Evolutionary Strategies. IJCSNS International Journal of Computer Science and Network Security, ISSN: 1738-7906, Vol. 10 No. 6 pp. 325-332 June, 2010.

[13] M. Merzougui, A. EL Allaoui, M. Nasri, M. EL Hitmy and $\mathrm{H}$. Ouariachi. Unsupervised classification using evolutionary strategies approach and the Xie and Beni criterion. IJAST International Journal of Advanced Science and Technology, ISSN: 2005-4238, Vol. 19, pp 43-58 June, 2010.
[14] Ammor.O, Raiss.N et Slaoui.K "Détermination du nombre optimal de classes présentant un fort degré de chevauchement " Revue Modulad $N^{\circ} 37$, pp 31-42, 2007.

[15] G.Palubinskas, X. Descombes et F. Kruggel. " An unsupervised clustering method using the entropy minimization. " In ICPR, pp. 1816-1818, Australia Août 1998.

[16] Kim, D. J, Park, Y. W. and Park, D. J. "A novel validity index for determination of the optimal number of clusters", IEICE Trans. Inform.Syst.D-E84. 2, 281 -285,2001 